\title{
Focus. Intertexualité, exogenèse, avant-textualité : le modèle joycien
}

Daniel Ferrer

\section{(2) OpenEdition}

1 Journals

Édition électronique

URL : https://journals.openedition.org/genesis/5702

DOI : 10.4000/genesis.5702

ISSN : 2268-1590

Éditeur :

Presses universitaires de Paris Sorbonne (PUPS), Société internationale de génétique artistique littéraire et scientifique (SIGALES)

Édition imprimée

Date de publication : 15 décembre 2020

Pagination : 107-110

ISBN : 979-10-231-0704-3

ISSN : 1167-5101

\section{Référence électronique}

Daniel Ferrer, «Focus. Intertexualité, exogenèse, avant-textualité : le modèle joycien », Genesis [En

ligne], 51 | 2020, mis en ligne le 20 décembre 2021, consulté le 07 février 2022. URL : http:// journals.openedition.org/genesis/5702 ; DOI : https://doi.org/10.4000/genesis.5702 
I maginons que le titre d'Ulysse soit par exemple Un jour dans la vie de Leopold Bloom. Imaginons que Joyce n'ait donné aucune indication sur ses intentions. Imaginons enfin qu'aucun des manuscrits préparatoires n'ait été disponible. Aurions-nous découvert l'intertextualité homérique qui structure le livre, qui gauchit sa texture et qui gouverne tant de ses détails? Il est plus que probable que nous n'aurions pas saisi l'importance et l'ampleur du rapport que le texte de Joyce entretient avec L'Odyssée. Nous n'aurions certainement pas perçu la correspondance systématique des chapitres du roman avec des épisodes de l'épopée. Aurions-nous deviné que la scène du bordel est identifiée au séjour chez Circé (parce que les hommes y sont transformés en cochons)? Nous n'aurions en tout cas pas eu l'idée de trouver un lien entre la scène de la bibliothèque et Charybde et Scylla (parce que Stephen Dedalus navigue entre les écueils de l'aristotélisme et du platonisme). Nous aurions donc lu le texte différemment, une dimension importante nous aurait échappé. Imaginons maintenant que les notes préparatoires de Joyce sur Homère, ses schémas, ses tables de correspondances, soient soudain découverts, en même temps que le jeu d'épreuves témoignant de l'ajout d'allusions homériques cryptées jusqu'à la veille de la publication ${ }^{1}$. La mise au jour de cette exogenèse massive changerait à l'évidence notre façon de lire le roman. Dirait-on que cet événement fortuit aurait créé cette intertextualité massive ou qu'elle l'aurait révélée ? Si l'intertextualité est bien un phénomène de lecture, n'est-on pas obligé de dire qu'elle n'existe pas, même à l'état latent ${ }^{2}$, tant qu'elle n'a pas été lue ${ }^{3}$ ?

Nous pouvons trouver chez Joyce de quoi creuser cette question sans avoir à nous appuyer sur des hypothèses aussi contrefactuelles. Précisons tout d'abord qu'il ne s'agit pas des allusions ésotériques, par exemple à d'obscures sources médiévales, que Joyce s'est complu à multiplier

\section{Intertexualité, exogenèse, avant-textualité : le modèle joycien}

\author{
Daniel Ferrer
}

dans Ulysse comme dans Finnegans Wake. Les critiques joyciens ont relevé le défi, faisant paraitre une multitude de travaux rivalisant d'érudition pour élucider ces allusions. Il ne s'agit pas non plus des déformations burlesques qui rendent presque méconnaissables les sentences les plus célèbres : «trespassing on the space question where even michelangelines fool to dread» ( $F W 160.36-161.014)$ ou «A king off duty and a jaw for ever!» (FW 162.35). Il faut quelques instants pour saisir l'allusion à Pope («Fools rush in where angels fear to tread») ou à Keats («A thing of beauty is a joy for ever»), mais il ne s'agit généralement que d'un effet de retardement de la reconnaissance qui finit par survenir soudain comme un choc, accompagné d'un

1. Ce scénario n'a rien d'absurde : de nombreux et importants manuscrits de Joyce ont été découverts ces dernières années, modifiant substantiellement notre compréhension de la genèse d'Ulysse et de Finnegans Wake. Il est notamment apparu une page de notes prouvant que la recherche des correspondances homériques était plus précoce et plus approfondie qu'on ne pouvait le supposer au préalable. Voir Daniel Ferrer, «An Unwritten Chapter of Ulysses? Joyce's Notes for a "Lacedemon" Episode », dans James Joyce: Whence, Whither and How. Studies in Honour of Carla Vaglio, G. Cortese, G. Ferreccio, M. T. Giaveri et T. Prudente (dir.), Alexandrie, Edizioni dell'Orso, 2015, p. 362-377. Voir aussi le surgissement de la malle de manuscrits de Raymond Roussel, qui a bouleversé encore beaucoup plus radicalement ce qu'on pouvait savoir de la genèse de certains de ses livres. Cf. Revue de la Bibliothèque nationale, no 43, 1992.

2. Voir ce que dit Bergson sur le possible, qui ne devient tel qu'a posteriori : La Pensée et le Mouvant, Paris, Alcan, 1934, p. 20.

3. Selon Michael Riffaterre, «l'intertextualité est la perception, par le lecteur, de rapports entre une œuvre et d'autres » («La trace de l'intertexte», La Pensée, no 215, 1980, p. 4). Il précise avec fermeté : «Phénomène textuel, l'intertextualité est immédiatement observable», «Avant-texte et littérarité», Genesis, no 9, p. 9-27, 1996 (en ligne sur persee.fr).

4. La (presque) totalité des éditions reprenant la même pagination, on indique ainsi les références : $F W 160.36$ signifie la ligne 36 de la page 160 de Finnegans Wake. 
sentiment d'inquiétante étrangeté, d'autant plus fort qu'aura été poussée la défamiliarisation de ce qui constitue notre intimité culturelle. Ce temps d'hésitation, que Joyce écrit «hesitency» au lieu de «hesitancy», sous le couvert d'une allusion au faussaire Pigott qui fut confondu au moyen de cette faute d'orthographe, est constitutif d'une stratégie joycienne de la différance généralisée :

Si je donne tout de suite tout, je perds mon immortalité. J'ai mis là tant d'énigmes et rébus qu'ils occuperont les professeurs à discuter pendant des siècles sur ce que j'ai voulu dire et c'est le seul moyen de s'assurer l'immortalité5.

On voit bien, à travers cette confidence de Joyce, que ce qui se refuse provisoirement doit se révéler dans un futur indéterminé dont on se rapprochera asymptotiquement par un travail d'élucidation. Et quand bien même un reste demeurerait inaccessible, ce qui est important, bien plus que l'identification précise de l'intertexte, c'est la perturbation, que Riffaterre appelle «agrammaticalité » ou «catachrèse », provoquée par l'importation dans le texte d'un élément exogène (tel le «e» du faussaire dans «hesitency»), le «connecteur», qui vient perturber le système indigène et invoque le fantôme d'un intertexte qui le surdétermine, même si l'on n'est pas à même de l'identifier précisément 6 .

Ce qui est véritablement problématique, c'est une relation qu'il est impossible de percevoir sans recours aux manuscrits et dont on trouve la trace dans les carnets de Finnegans Wake ${ }^{7}$. On songe à ce personnage de Lucky Luke qui aimait à citer Shakespeare, mais choisissait des passages parfaitement insignifiants : «Voilà qui est parfait », Roméo et Juliette, acte II, scène 4; «C'est bien », Hamlet, acte IV, scène $3^{8}$. S'il choisissait d'utiliser ces citations, c'est bien évidemment pour leur provenance et non pour leur contenu. Il semble que Joyce fasse de même, mais lui se garde bien d'indiquer la référence, ce qui fait que la «citation» reste indiscernable à cause de sa banalité. Cette banalité tient soit, comme pour le Shakespeare de Lucky Luke, au choix de la citation, qui est tout sauf caractéristique (ainsi les termes prélevés chez Freud n'appartiennent pas au vocabulaire de la psychanalyse), soit à sa provenance. À côté de son goût, mentionné plus haut, pour les allusions ésotériques, réservées à une élite culturelle, comme le Livre des Morts égyptien, Joachim de Flore ou Giordano Bruno, Joyce s'est de plus en plus intéressé au discours commun. Il s'immerge dans le flot des paroles imprimées dans les journaux et les prospectus, les manuels et les encyclopédies, et va puiser abondamment dans cette «littérature» dont personne ne garde le souvenir, dans ce discours de seconde ou de troisième main, trop commun pour être identifiable. Ce qui importe dans ces prélèvements qui s'étalent sur des milliers de pages de carnets avant d'être repris pour être injectés ponctuellement dans Finnegans Wake, c'est moins leur substance que leur appartenance à ce bruit de fond, à cette rumeur indistincte de la civilisation écrite dans laquelle nous baignons, où les grands textes ne sont plus qu'une voix parmi tant d'autres, de même que le sol sur lequel nous marchons est composé de la poussière de nos prédécesseurs, grands hommes et anonymes, indissociablement mêlés. Qu'en est-il, dans ce cas de l'agrammaticalité riffaterrienne? Elle est certes présente, comme à chaque fois qu'un élément exogène est introduit dans un contexte, mais elle est faible au point d'être indiscernable, à cause de la banalité des éléments importés, et surtout à cause de l'omniprésence des agrammaticalités qui affectent chaque mot, voire chaque lettre de Finnegans Wake, au point qu'il n'y a plus vraiment de contexte de référence par rapport auquel peut être appréciée la grammaticalité. Il est donc indispensable de passer par les carnets de notes de Joyce pour percevoir ce système d'allusions inouïes.

Voici donc un cas où l'intertextualité ne peut fonctionner sans recours aux documents exogénétiques. On peut penser que ce cas (très) particulier du rapport entre le texte et son avant-texte est généralisable : ce qui est vrai des notes de lecture de Joyce est vrai de chaque variante écartée dans les manuscrits, de chaque version supplantée par une autre. Chaque modification laisse une trace dans le texte (une

5. Rapporté par Jacques Benoist-Méchin. Richard Ellmann, James Joyce, t. II, trad. A. Cœuroy et M. Tadié, Paris, Gallimard, 1987, p. 154.

6. Michael Riffaterre, «L'intertexte inconnu », Littérature, no 2, 41, 1981 et «La trace de l'intertexte», art. cit.

7. Pour une présentation de ces carnets, je me permets de renvoyer le lecteur à un article ancien : «Les carnets de Joyce, avant-textes limites d'une œuvre limite», Genesis, no 3, 1993, p. $45-61$ (en ligne sur persee.fr). Voir aussi «"L'oreille paradigmatique". Les listes dans les manuscrits de James Joyce», Genesis, no 47, 2018, p. 81-91 (en ligne sur openedition.org). 8. Morris, Jesse James, Dargaud, 2013, p. 7. 


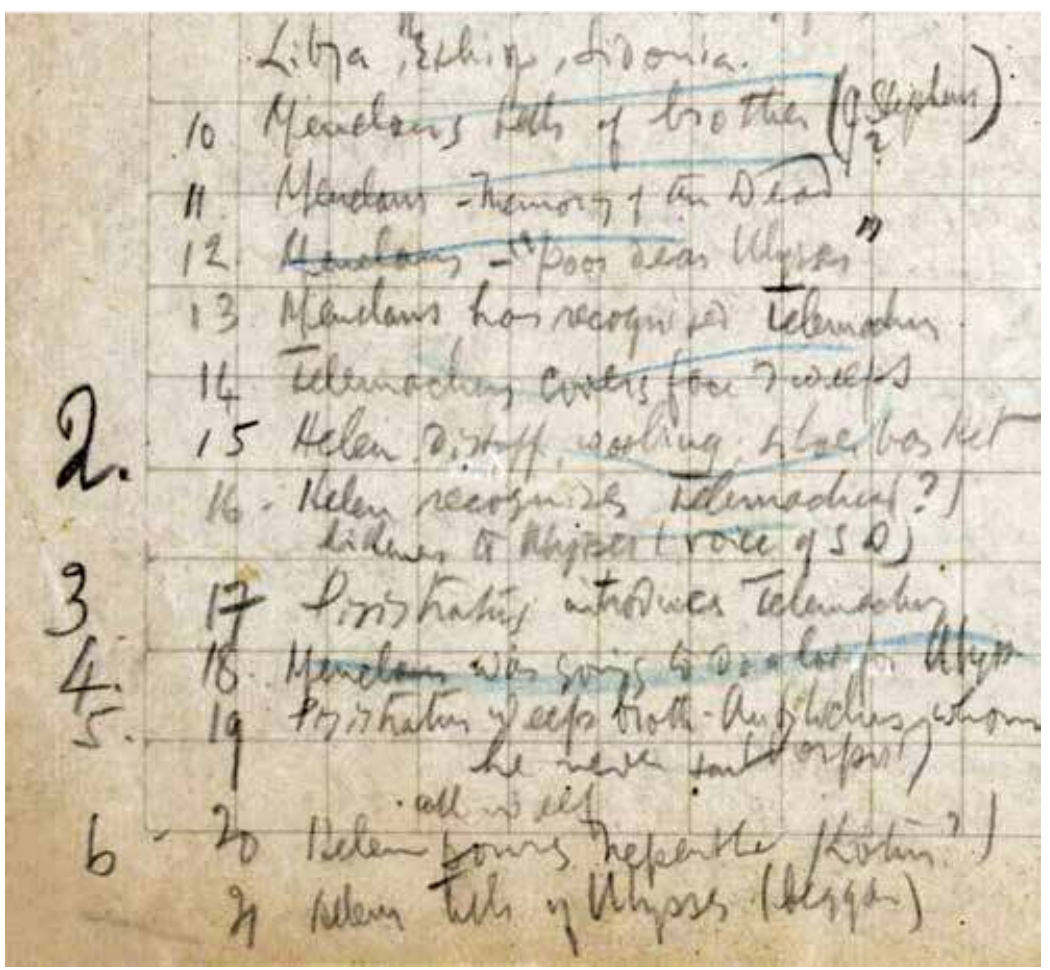

Fig. 1 : Détail des notes prises par Joyce sur L'Odyssée dans la traduction de Butcher et Lang (1879), avec, entre parenthèses, des amorces de transposition.

(C) National Library of Ireland, The Joyce Papers 2002, Cahier II. ii.1.a, dernière page. Avec l'aimable autorisation de la National Library of Ireland

agrammaticalité si l'on veut) puisque la modification est venue bouleverser le système préexistant par l'intrusion d'un élément qui n'existait pas au stade antérieur. Le texte contient donc une allusion silencieuse au changement qui est intervenu, allusion dont on peut percevoir l'écho diffus, mais qu'il est impossible de préciser sans avoir recours à l'avant-texte. C'est ce mécanisme qui est à la base de la «mémoire du contexte ${ }^{9}$, c'est lui qui constitue la meilleure légitimation des efforts des généticiens.

9. Voir Daniel Ferrer, «La toque de Clementis : rétroaction et rémanence dans les processus génétiques », Genesis, no 6, «Enjeux critiques », 1994 (en ligne sur persee.fr) et Logiques du brouillon : modèles pour une critique génétique, Paris, Seuil, 2011, p. 109-113. 
DANIEL FERRER est chercheur à l'ITEM (ENS-CNRS). Il a écrit des livres et des articles sur Joyce, Woolf, Faulkner, Poe, Stendhal, Flaubert, Zola, Barthes, Cixous, sur la peinture, les humanités numériques, la génétique cinématographique et la théorie de la critique génétique. Ouvrages récents : Logiques du brouillon. Modèles pour une critique génétique (Seuil, coll. «Poétique», 2011); Renascent Joyce (Gainesville, University Press of Florida, 2013) et l'édition de Brouillons d'un baiser : premiers pas vers Finnegans Wake (Gallimard, coll. «Du monde entier», 2014).

ferrerdan@me.com

\section{Intertexualité, exogenèse, avant-textualité : le modèle joycien}

Cette notule reprend la question des rapports entre intertextualité et exogenèse à partir d'exemples empruntés à Joyce. Comment définir une relation qui est totalement imperceptible dans le texte publié, mais qui a joué un rôle important dans sa genèse ? Ce type d'allusion inaudible peut nous servir de modèle pour penser le rapport du texte à son avant-texte.

This short note revisits the question of the connection between intertextuality and exogenesis in examples pulled from Joyce's manuscripts. How can we define something that played an important role in genesis but that is entirely absent in the published text? This kind of imperceptible allusion can be used as a model to understand the relation between the text and its "avant-texte".

Dieses Notule greift die Frage nach dem Verhältnis von Intertextualität und Exogenese anhand von Joyce entlehnten Beispielen auf. Wie definiert man eine Beziehung, die in dem veröffentlichten Text völlig unmerklich ist, die aber bei seiner Entstehung eine wichtige Rolle gespielt hat? Diese Art von unhörbarer Anspielung kann als Modell für das Nachdenken über das Verhältnis des Textes zu seinem Vorwort dienen.
Esta nota retoma la cuestión de las relaciones entre intertextualidad y exogénesis a partir de ejemplos tomados de Joyce. ¿Cómo definir una relación que es totalmente imperceptible en el texto publicado, pero que ha jugado un papel importante en su génesis? Este tipo de alusión inaudible puede servirnos de modelo para considerar la relación del texto con su pre-texto.

Esta breve nota retoma a questão das relações entre intertextualidade e exogénese a partir de exemplos dos manuscritos de Joyce. Como definir uma relação totalmente impercetível no texto publicado, mas que desempenhou um papel importante na sua génese? Este tipo de alusão inaudível pode servir-nos de modelo para pensar a relação do texto com o seu ante-texto.

Questa breve nota riprende la questione dei rapporti tra intertestualità ed esogenesi partendo da esempi tratti da Joyce. Come definire una relazione che è totalmente impercettibile nel testo pubblicato ma che ha avuto un ruolo importante nella sua genesi? Questo tipo d'allusione impalpabile può servirci da modello per pensare il rapporto del testo con il suo avantesto. 